Arch Virol (1989) 104: 187-196

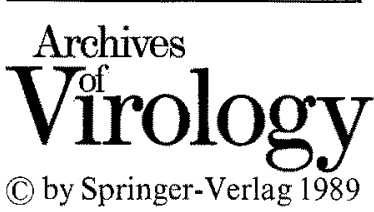

\title{
Virus strain specificity of challenge immunity to coronavirus
}

\author{
S. W. Barthold and Abigail L. Smith \\ Section of Comparative Medicine, Yale University School of Medicine, New Haven, \\ Connecticut, U.S.A.
}

Accepted December 5, 1988

\begin{abstract}
Summary. The resistance of immunized mice to challenge with the same or a different strain of mouse hepatitis virus (MHV) was examined as a model of challenge immunity to coronavirus infection. Genetically susceptible BALB/ cByJ mice were given an intranasal immunizing infection of respiratory-type MHV-JHM, MHV-S, or enterotropic MHV-Y. Control mice were sham-immunized with sterile tissue culture fluid. Recovered mice were challenged intranasally with MHV-JHM, MHV-S or sterile tissue culture fluid at 30 days after immunization. Resistance to challenge inoculation was evaluated in groups of mice at 4 and 30 days after challenge. At 4 days, the prevalence of MHV lesions in nose and liver was tabulated and MHV titers in liver were determined. At 30 days, the prevalence of residual brainstem spongiform lesions was tabulated and serum antibody to MHV-JHM and MVH-S was quantified by enzyme immunoassay. Mice immunized with MHV-JHM or MHV-S resisted challenge with the MHV homotype, but MHV-S-immunized mice were fully susceptible to challenge with MVH-JHM. Mice immunized with enterotropic MHV-Y were only partially protected against challenge with antigenically related, but biologically different MHV-S. Serum antibody responses to MHV supported these observations. These data indicate that challenge immunity to coronaviruses is strong, but highly virus strain-specific.
\end{abstract}

\section{Introduction}

Coronaviruses are common respiratory and enteric pathogens of a variety of birds and mammals, including man. About $15 \%$ of common colds in man are caused by coronaviruses [25]. The frequency of repeated colds in a single individual and the frequency of association with a coronaviral etiology suggest that an individual can be repeatedly infected with coronaviruses. If so, host immunity is ineffective, or effectively directed toward virus strain-specific antigens. Mouse hepatitis virus (MHV) is an extensively studied coronavirus that might provide insight into the mechanism of this phenomenon. This virus is represented by many different strains that vary biologically and possess minor 
antigenic differences. Different MHV strains display primary tropism for either the upper respiratory or enteric mucosa [1]. They share complex antigenic interrelationships, with cross-reactivity among themselves, as well as with coronaviruses of rats, man (OC 43 group), cattle and pigs (hemagglutinating encephalomyelitis virus) [16].

Like other coronaviruses, the MHV virion consists of 3 major antigenic components: nucleoprotein $(\mathrm{N})$, membrane glycoprotein $(\mathrm{E} 1)$, and peplomeric glycoprotein $(\mathrm{E} 2) . \mathrm{N}$ and $\mathrm{E} 1$ antigens are highly conserved among MHV strains, but $\mathrm{E} 2$ is polymorphic and responsible for MHV strain-specific antigenic identity $[13,24]$. E 2 is the primary target for neutralizing antibody $[9,10,13]$ and challenge immunity of mice to $\mathrm{MHV}$ can be induced by vaccination with whole virus or peplomers, but not membrane or nucleoprotein subcomponents [18]. This would suggest that effective host immunity to virus challenge is directed toward the most strain-specific antigenic epitopes of the virion. The polymorphism of $\mathrm{E} 2$ possibly represents an effective survival strategy of the virus to evade immune clearance from a host population. Indeed, E 2 antigenic changes can be readily selected by escape from neutralization with monoclonal antibodies in vitro [11].

The purpose of this study was to examine the specificity of host challenge immunity against the same virus or against virus strains that differed biologically or antigenically from the immunizing strain, using an MHV model. Intranasal (i.n.) immunization and challenge with live virus were chosen to mimic a natural route of exposure.

\section{Materials and methods}

\section{Experimental plan and rationale}

The resistance of previously immunized mice to challenge with the same or a different strain of MHV was examined following i.n. immunization and challenge. Three MHV strains were used. MHV-JHM and MHV-S are antigenic heterotypes by serum neutralization $[8,15]$, but are biologically similar, since they both have primary upper respiratory tropism, with secondary tropism for liver and brain [5-7]. MHV-Y is closely related to MHV-S antigenically, but differs biologically from MHV-S being primarily enterotropic with minimal tropism for liver and brain $[6,8]$. BALB/cByJ mice were used because of their genetic susceptibility to the MHV strains used $[2,7]$. Immunization was accomplished by i.n. inoculation of 3 week old mice with live MHV-JHM, MHV-S, MHV-Y or sterile tissue culture fluid (TCF). Mice were held for 30 days, an interval in which mice recover from MHV infection after i.n. inoculation $[2,7]$, and were then challenged i.n. with MHVJHM, MHV-S or TCF.

Resistance to challenge was evaluated by microscopic examination of nose at 4 days after challenge. This site is the primary mucosal target of MHV-JHM and MHV-S [7, 22]. The ability of challenge virus to spread to secondary target organs (liver and brain) was also assessed as an index of susceptibility. Dissemination of MHV was measured by microscopic examination and virus titration of liver at 4 days after challenge. Four days is a peak interval for acute MHV disease following i.n. inoculation [7]. MHV-JHM and MHV-S also spread directly to brain from nose via olfactory nerves, leaving a high prevalence of residual brainstem spongiosis at 30 days after i.n. inoculation $[3,4]$. Thus, brains 
were examined at 30 days after challenge. Sera from mice killed at 30 days after challenge were tested for antibody to MHV-S and MHV-JHM. Treatment groups and the number of mice in each are summarized in Tables 1-3 and Figs. 1 and 2.

\section{Mice}

Three-week-old inbred BALB/cByJ and adult pregnant outbred CR 1: CD 1 BR Swiss mice were obtained from the Jackson Laboratory, Bar Harbor, ME, and Charles River Laboratories, Portage, MI, respectively. Mice from both sources were serologically verified as MHV-free. Mice were shipped in filtered boxes and transferred upon arrival into autoclaved Micro-isolator containment cages (Lab Products, Maywood, NJ) containing wood shavings, food (Purina Laboratory Chow, Ralston Purina Company, St. Louis, MO) and water. Pathogen-free sentinel mice were maintained in adjacent, open cages and tested periodically for serum MHV antibody to monitor for inadvertant contamination of the room with wildtype or experimental MHV strains.

Sera of mice killed on day 4 after challenge (34 days after immunization) or at the time of challenge were tested for MHV antibody to verify immunization status. Mice were selected randomly from treatment groups and were killed with carbon dioxide gas, followed by cardiac exsanguination. Sera and liver were stored at $-70^{\circ} \mathrm{C}$ until tested for antibody or virus. Tissues were fixed in $10 \%$ neutral buffered formalin, $\mathrm{pH} 7.2$, paraffin embedded, and processed by routine histological methods.

\section{Virus}

MHV-JHM and MHV-S were obtained from the American Type Culture Collection, Rockville, MD. MHV-JHM was subsequently passaged twice in NCTC 1469 cells, once in adult BALB/cByJ mouse liver and once in $17 \mathrm{Cl} 1$ cells. MHV-S was passaged in NCTC 1469 cells. MHV-Y was obtained from an outbreak in infant mice and maintained by infant mouse passage [8]. Aliquots of each virus inoculum were frozen at $-70^{\circ} \mathrm{C}$ until used. Mice were inoculated i.n. with $20 \mu \mathrm{l}$ of cell-free culture fluid containing approxiamtely $10^{3}$ $\mathrm{TCID}_{50}$ of MHV. Virus in liver was titrated by intracerebral inoculation of infant Swiss mice with serial 10 -fold $\mathrm{w} / \mathrm{v}$ dilutions of tissue homogenates, as previously described [7], and expressed as $\log _{10} \mathrm{LD}_{50} /$ gram.

\section{Serology}

Serum MHV antibody titers were determined using an enzyme immunoassay (EIA) with formalin-fixed MHV-JHM- or MHV-S-infected $17 \mathrm{Cl} 1$ cells as antigen [7,21]. Horseradish peroxidase-conjugated goat anti-mouse IgG (Tago, Burlingame, CA) and ABTS substrate (Kirkegaard and Perry Laboratories, Gaithersburg, MD) were used in these tests. To verify immune status at the time of challenge or to monitor for inadvertant MHV contamination of treatment groups, sera were tested for MHV antibody by indirect immunofluorescence assay [19].

\section{Statistical analysis}

Virus titers were compared between groups with Student's unpaired t test. Prevalence data were compared by Chi-square analysis.

\section{Results}

Based upon nose and liver lesion prevalence as well as MHV titers in liver during the acute phase of challenge infection (day 4), mice resisted MHV challenge when immunized with the same MHV strain, but were susceptible to 
MHV challenge when immunized with a different MHV strain (Table 1, Figs. 1 and 2). Immunized mice challenged with the MHV homotype had virtually no nose or liver lesions compared to sham-immunized mice. MHV-JHM and MHV$\mathrm{S}$ immunized mice had significantly lower virus titers in their livers compared to sham-immunized mice challenged with MHV-JHM or MHV-S ( $\mathrm{P} \leqslant 0.001$ and $P \leqslant 0.05$, respectively). Mice immunized with $\mathrm{MHV}-\mathrm{S}$, then challenged with MHV-JHM, developed a similar prevalence of nose and liver lesions and similar virus titers in liver compared to sham-immunized mice, indicating no resistance to challenge. Mice immunized with enterotropic MHV-Y, then challenged with antigenically related, but biologically different $\mathrm{MHV}-\mathrm{S}$, had fewer nose lesions $(\mathrm{P} \leqslant 0.01)$ but a similar prevalence of liver lesions and comparable liver virus titers compared to sham-immunized, MHV-S challenged mice. Conversely, nose lesion prevalence did not differ from MHV-S immunized, MHV-S challenged mice, but liver lesion prevalence $(P \leqslant 0.01)$ and liver virus titers $(P \leqslant 0.01)$ were significantly higher.

The presence of brain lesions at 30 days after challenge also reflected virus type-specific immunity (Table 2). Immunized mice developed a low prevalence of or no spongiform brain lesions when challenged with the antigenically homotypic MHV strain, compared to sham-immunized controls. In contrast to liver, brains of mice immunized with enterotropic MHV-Y were protected from challenge with antigenically similar, but biologically different MHV-S. Brain lesions seen at 30 days after challenge were due to the challenge, rather than the immunizing infection, since mice that were immunized with TCF, MHV-JHM, MHV-S or MHV-Y, then sham-challenged had no detectable spongiform brain lesions remaining at 30 days after sham challenge.

Table 1. Prevalence of acute MHV lesions in primary (nose) and secondary (liver) target organs at 4 days after intranasal challenge inoculation ( 34 days after immunizing infection)

\begin{tabular}{|c|c|c|c|c|c|c|c|}
\hline \multicolumn{3}{|c|}{ Treatment group } & \multicolumn{2}{|c|}{ Prevalence $^{\mathfrak{a}}$} & \multicolumn{3}{|c|}{ Chi square probability ${ }^{b}$} \\
\hline Number & $\begin{array}{l}\text { MHV } \\
\text { immunogen }\end{array}$ & $\begin{array}{l}\text { MHV } \\
\text { challenge }\end{array}$ & Nose & Liver & Comparison $^{\mathrm{c}}$ & Nose & Liver \\
\hline 1. & $\theta^{d}$ & JHM & $19 / 24$ & $25 / 25$ & & & \\
\hline 2. & JHM & JHM & $0 / 10$ & $0 / 10$ & 1 vs. 2 & 0.001 & 0.001 \\
\hline 3. & $\theta$ & $\mathrm{S}$ & $14 / 20$ & $8 / 10$ & & & \\
\hline 4. & $\mathrm{~S}$ & $\mathrm{~S}$ & $0 / 10$ & $0 / 10$ & 3 vs. 4 & 0.05 & 0.05 \\
\hline 5. & $\mathrm{~S}$ & JHM & $10 / 11$ & $11 / 11$ & $\begin{array}{l}1 \text { vs. } 5 \\
2 \text { vs. } 5\end{array}$ & $\begin{array}{l}\text { n.s. } \\
0.001\end{array}$ & $\begin{array}{l}\text { n.s. } \\
0.001\end{array}$ \\
\hline 6. & $\mathrm{Y}$ & $\mathrm{S}$ & $2 / 8$ & $5 / 10$ & $\begin{array}{l}3 \text { vs. } 6 \\
4 \text { vs. } 6\end{array}$ & $\begin{array}{l}0.01 \\
\text { n.s. }\end{array}$ & $\begin{array}{l}\text { n.s. } \\
0.01\end{array}$ \\
\hline
\end{tabular}

a Number positive/number examined

b Probability, $\mathrm{P} \leqslant ; n . s$. not significant

c Chi square comparison, group numbers compared

${ }^{d} \theta$ Sham inoculated (tissue culture fluid) 


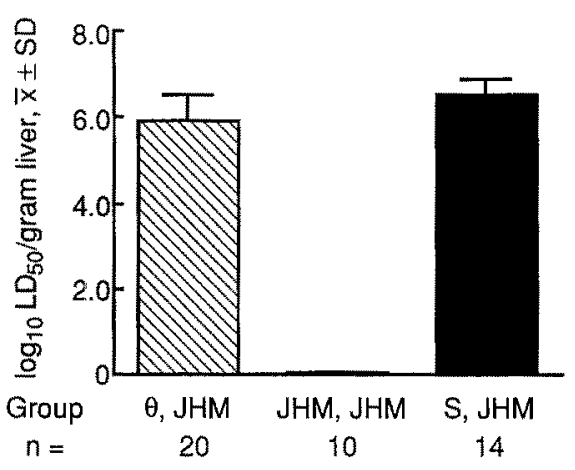

Fig. 1

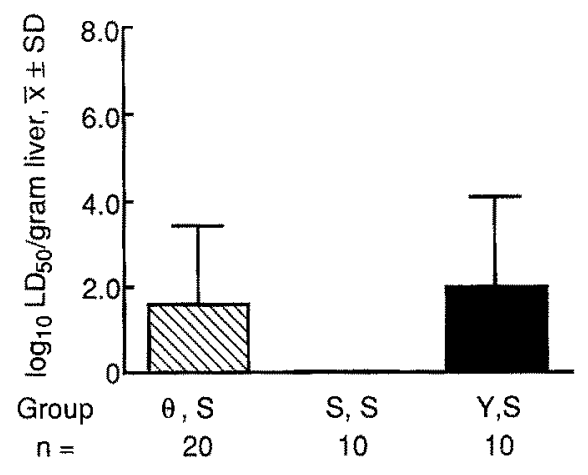

Fig. 2

Fig. 1. MHV titers in livers of BALB/CByJ mice 4 days after intranasal challenge with MHV-JHM. Groups include sham-immunized/MHV-JHM-challenged $(\theta, J H M)$; MHVJHM-immunized/MHV-JHM-challenged $(J H M, J H M)$ and MHV-S-immunized/MHVJHM-challenged $(S, J H M)$ mice

Fig. 2. MHV titers in livers of $B A L B / C B y J$ mice 4 days after intranasal challenge with MHV-S. Groups include sham-immunized/MHV-S-challenged $(\theta, S)$; MHV-S-immunized/ MHV-S challenged $(S, S)$ and MHV-Y-immunized/MHV-S-challenged $(Y, S)$ mice

Table 2. Prevalence of spongiform MHV lesions in brain at 30 days after intranasal challenge inoculation ( 60 days after immunizing infection)

\begin{tabular}{|c|c|c|c|c|c|}
\hline \multicolumn{3}{|c|}{ Treatment group } & \multirow[t]{2}{*}{ Prevalence $^{a}$} & \multicolumn{2}{|l|}{ Chi square } \\
\hline Number & $\begin{array}{l}\text { MHV } \\
\text { immunogen }\end{array}$ & $\begin{array}{l}\text { MHV } \\
\text { challenge }\end{array}$ & & Comparison $^{b}$ & Probability $^{c}$ \\
\hline 1. & $\theta^{\mathrm{d}}$ & JHM & $10 / 13$ & & \\
\hline 2. & JHM & JHM & $1 / 11$ & 1 vs. 2 & 0.001 \\
\hline 3. & $\theta$ & $\mathbf{S}$ & $15 / 23$ & & \\
\hline 4. & $\mathrm{~S}$ & $S$ & $1 / 13$ & 3 vs. 4 & 0.001 \\
\hline 5. & $\mathrm{~S}$ & JHM & $9 / 17$ & $\begin{array}{l}1 \text { vs. } 5 \\
2 \text { vs. } 5\end{array}$ & $\begin{array}{l}\text { n.s. } \\
0.05\end{array}$ \\
\hline 6. & $\mathrm{Y}$ & $S$ & $1 / 13$ & $\begin{array}{l}3 \text { vs. } 6 \\
4 \text { vs. } 6\end{array}$ & $\begin{array}{l}0.001 \\
\text { n.s. }\end{array}$ \\
\hline 7. & $\theta$ & $\theta$ & $0 / 19$ & & \\
\hline 8. & JHM & $\theta$ & $0 / 8$ & & \\
\hline 9. & $\mathrm{~S}$ & $\theta$ & $0 / 10$ & & \\
\hline 10. & $\mathrm{Y}$ & $\theta$ & $0 / 10$ & & \\
\hline
\end{tabular}

${ }^{a}$ Number positive/number examined

${ }^{b}$ Chi square comparison, group numbers compared

${ }^{c}$ Probability, $\mathrm{P} \leqslant ;$ n.s. not significant

${ }^{d} \theta$ Sham inoculated (tissue culture fluid) 
Seroconversion and antibody titers to MHV-JHM and MHV-S reflected the antigenic experience of immunized and challenged mice at 30 days after challenge (Table 3). Sera from sham-inoculated mice (group 7) did not react with either antigen. Sera from mice inoculated only with MHV-JHM 30 and/ or 60 days prior to bleeding (groups 1,2 , and 8 ) reacted with both homologous and heterologous antigen in the EIA. Sera from mice inoculated only with MHV-S groups 3, 4, and 9) uniformly reacted with homologous antigen, but not all sera reacted with MHV-JHM. Among these groups, there was not an obvious correlation between high titer to MHV-S and reactivity with MHVJHM. Sera from mice immunized with MHV-Y and challenged with MHV-S (group 6) reacted with MHV-S to the same extent as sera from mice that were immunized but not challenged (group 10). However, sera from group 6 mice reacted relatively poorly with $\mathrm{MHV}-\mathrm{JHM}$ anigen. Sera from mice immunized with MHV-S and challenged with MhV-JHM (group 5) reacted more uniformly and to substantially higher titer with MHV-JHM than sera from mice immunized with MHV-S but not challenged (group 9).

Table 3. Serum antibody EIA reactivity of mice to MHV-JHM and MHV-S, 60 days after primary immunizing infection and 30 days after challenge inoculation

\begin{tabular}{|c|c|c|c|c|c|c|}
\hline \multicolumn{3}{|c|}{ Treatment group } & \multicolumn{2}{|c|}{ MHV-JHM antigen } & \multicolumn{2}{|c|}{ MHV-S antigen } \\
\hline Number & $\begin{array}{l}\text { MHV } \\
\text { immunogen }\end{array}$ & $\begin{array}{l}\text { MHV } \\
\text { challenge }\end{array}$ & Prevalence $^{\mathrm{a}}$ & Titer $^{\mathrm{b}}$ & Prevalence & Titer \\
\hline 1. & $\theta^{c}$ & JHM & $13 / 13$ & 4,672 & $13 / 13$ & 2,832 \\
\hline 2. & JHM & JHM & $16 / 16$ & 8,704 & $16 / 16$ & 9,280 \\
\hline 3. & $\theta$ & $\mathrm{S}$ & $7 / 19$ & 100 & $23 / 23$ & 3,744 \\
\hline 4. & $S$ & $\mathrm{~S}$ & $8 / 13$ & 69 & $13 / 13$ & 2,960 \\
\hline 5. & $S$ & JHM & $17 / 17$ & 752 & $17 / 17$ & 23,296 \\
\hline 6. & $Y$ & $\mathrm{~S}$ & $12 / 13$ & 492 & $13 / 13$ & 1,600 \\
\hline 7. & $\hat{\theta}$ & $\theta$ & $0 / 19$ & & $0 / 19$ & \\
\hline 8. & JHM & $\theta$ & $8 / 8$ & 2,800 & $8 / 8$ & 1,440 \\
\hline 9. & $\mathrm{~S}$ & $\theta$ & $4 / 10$ & 75 & $10 / 10$ & 320 \\
\hline 10. & $Y$ & $\theta$ & $10 / 10$ & 3,500 & $10 / 10$ & 1,600 \\
\hline
\end{tabular}

a Number with reactivity at serum dilution $\geqslant 1: 50 /$ number tested

${ }^{b}$ Reciprocal of geometric mean for positive sera

${ }^{c} \theta$ Sham inoculated (tissue culture fluid)

\section{Discussion}

Challenge resistance of immunized mice appears to depend upon both the antigenic type and the biotype of the MHV strains used to immunize and challenge. Mice immunized with respiratory-type MHV-JHM or MHV-S resisted challenge with the antigenic MHV homotype, but were as susceptible as naive mice to challenge with the antigenic heterotype. Mice immunized with 
MHV-JHM and challenged with MHV-S were not examined because of the extremely high mortality due to encephalitis among mice infected i.n. with MHVJHM. All surviving MHV-JHM immunized mice were used to test resistance to MHV-JHM. Mice immunized with enterotropic MHV-Y were only partially protected against challenge with biologically different respiratory-type MHV$\mathrm{S}$, despite their close antigenic relatedness by serum neutralization. Mice immunized with MHV-S and challenged with MHV-Y were not studied because MHV-Y has minimal tropism for brain or liver, which were the target organs used to measure challenge resistance.

Antigenic relationships among different MHV strains are complex and difficult to define. Nucleocapsid and E1 membrane antigens are substantially conserved among MHV strains, while E 2 peplomeric antigens are highly variable, although there are conserved E 2 epitopes as well [13]. Neutralizing antibody is directed primarily at E2 antigens [10], and is used as a measure of antigenic variation among $\mathrm{MHV}$ strains $[8,15]$. Although the immune response is directed toward N, E 1 and $\mathrm{E} 2$ antigens, effective challenge immunity appears to be E2-mediated. Resistance to intraperitoneal (i.p.) challenge with MHV can be induced against the MHV homotype by prior vaccination with inactivated whole virus or peplomers, but not with virus membrane or nucleoprotein subcomponents [18]. Challenge resistance to MHV could be conferred passively with two of three neutralizing monoclonal antibodies to epitopes of $E 2$, but not non-neutralizing antibodies to E 2, E 1 or $\mathrm{N}$ antigens [9]. These observations tend to support the current findings of virus strain-specific challenge immunity. Further support includes a study in which mice immunized i.p. with live MHV1 were not protected against i.p. challenge with MHV-3 [12]. On the other hand, mice immunized i.n. with live MHV-S resisted a normally lethal i.p. challenge with MHV-2, but MHV-S antibody neutralizes MHV-2, suggesting E 2 cross-reactivity between these strains [23]. Mice immunized i.n. with MHVJHM were also protected against death when challenged i.n. with MHV-3 [14]. At least one E2 epitope is shared between MHV-JHM and MHV-3 [13], possibly explaining the cross-protection. In the present study, MHV-JHM and MHV-S were chosen, since they appear to be antigenically disparate by serum cross-neutralization [15]. Under these circumstances, strain specificity of challenge resistance was apparent.

Although MHV-Y is closely related to MHV-S by serum neutralization [8], immunization with MHV-Y conferred only partial protection against MHVS. Despite the close antigenic relationship, MHV-S and MHV-Y differ significantly biologically. MHV-Y is restrictively enterotropic, with little or no hepatoor neurotropism $[2,6,8]$ while MHV-S has primary respiratory tropism with secondary hepato- and neurotropism [4-6, 22]. Mice immunized with MHV$\mathrm{Y}$ resisted MHV-S challenge at the nasal mucosa and brain, but not liver. This could be explained by the separate pathways by which brain and liver become infected with MHV in adult immunocompetent mice. Following i.n. inoculation, MHV can extend directly into brain via olfactory nerves after replicating initially 
in nasal olfactory mucosa; however, liver is infected by viremic dissemination [3]. Intranasal alpha/beta interferon treatment can block MHV replication in nasal mucosa and thereby appears to prevent spread of virus to brain, although viremic dissemination to liver still occurs [20]. Likewise, oral inoculation of MHV circumvents the nose-to-brain pathway, but results in hepatitis [3]. Immunization with enterotropic MHV-Y may induce an immune response that is localized to mucosal surfaces, thus protecting nose and brain, but not liver upon MHV-S challenge. The effect could also be a reflection of the close, but not exact, antigenic relatedness of the two MHV strains.

The serological responses of mice that were immunized and/or challenged with the MHV strains used in this study raise some interesting questions about the antigens used for detection in the EIA. Sera from mice immunized and/or challenged with MHV-JHM reacted with both homologous and MHV-S antigen. In contrast, sera from mice immunized and/or challenged with MHV-S reacted more uniformly and to substantially higher titer with the homologous antigen. Thus, the MHV-S antigen is more cross-reactive than the MHV-JHM antigen. Infected cell monolayers in which $50-75 \%$ of the cells are fused are used as antigen in the EIA [21]. Syncytia formation by MHV relies on the presence of the E 2 glycoprotein which is also the protein against which neutralizing (strain-specific) antibody is directed. The MHV-JHM stock used for $17 \mathrm{Cl} 1$ cell infection produces much larger syncytia than does the MHV-S stock. One explanation for the strain-specific reactivity with MHV-JHM antigen is that relatively higher concentrations of E 2 protein may be expressed by cells infected with this virus. Thus, the EIA may, under certain conditions, be more likely to detect neutralizing antibody than enzyme immunoassays that use immunobilized soluble antigen.

These results have demonstrated that mice immunized by i.n. inoculation of live virus are highly resistant to i.n. challenge with the same MHV strain, thus negating the hypothesis that host immunity to coronavirus is ineffective. On the other hand, results demonstrate that immunized mice remain fully susceptible to challenge with a different strain of coronavirus. This reinforces the hypothesis that effective host immunity is directed primarily toward the most variable $\mathrm{E} 2$ component of the virion, explaining susceptibility to repeated infections. Modification of E2, especially under selective immune pressure, may be a successful survival strategy of coronaviruses to evade clearance from immune populations. The high mutation frequency of RNA viral genomes, the high rate of MHV RNA recombination [17], the preferential selective pressure on $\mathrm{E} 2$ polymorphism $[17,24]$, and the large number of known MHV strains with minor antigenic differences [1] support this premise.

\section{Acknowledgements}

This work was supported by grant RR 02039 from the Division of Research Resources, National Institutes of Health, Bethesda, Maryland. The technical assistance of D. S. Beck and D.F. Winograd and the secretarial assistance of D. Crowder is gratefully acknowledged. 


\section{References}

1. Barthold SW (1986) Mouse hepatitis virus biology and epizootiology. In: Bhatt PN, Jacoby RO, Morse AC III, New AE (eds) Viral and mycoplasmal infections of laboratory rodents: effects on biomedical research. Academic Press, Orlando, FL, pp 571601

2. Barthold SW (1987) Host age and genotypic effects on enterotropic mouse hepatitis virus infection. Lab Anim Sci 37: 36-40

3. Barthold SW (1988) Olfactory neural pathway in mouse hepatitis virus nasoencephalitis. Acta Neuropathol 76: 502-506

4. Barthold SW, Beck DS, Smith AL (1986) Mouse hepatitis virus nasoencephalopathy is dependent upon virus strain and host genotype. Arch Virol 91: 247-256

5. Barthold SW, Smith AL (1983) Mouse hepatitis virus S in weanling Swiss mice following intranasal inoculation. Lab Anim Sci 33: 355-360

6. Barthold SW, Smith AL (1984) Mouse hepatitis virus strain-related patterns of tissue tropism in suckling mice. Arch Virol 81: 103-112

7. Barthold Sw, Smith AL (1987) Response of genetically susceptible and resistant mice to intranasal inoculation with mouse hepatitis virus JHM. Virus Res 7: 225-239

8. Barthold SW, Smith AL, Lord PFS, Bhatt PN, Jacoby RO, Main AJ (1982) Epizootic coronaviral typhlocolitis in suckling mice. Lab Anim Sci 32: 376-383

9. Buchmeier MJ, Lewicki HA, Talbot PJ, Knobler RC (1984) Murine hepatitis virus4(strain JHM)-induced neurologic disease is modulated in vivo by monoclonal antibodies. Virology 132: 261-270

10. Collins AR, Knobler RL, Powell H, Buchmeier MJ (1982) Monoclonal antibodies to murine hepatitis virus-4(strain JHM) define the viral glycoprotein responsible for attachment and cell-cell fusion. Virology $119: 358-371$

11. Dalziel RG, Lamper PW, Talbot PJ, Buchmeier MJ (1986) Site-specific alteration of murine hepatitis virus type 4 peplomer glycoprotein $\mathrm{E} 2$ results in reduced neurovirulence. J Virol 59: 463-471

12. Dick G, Niven J, Gledhill A (1956) A virus related to that causing hepatitis in mice (MHV). Br J Exp Pathol 27: 90-98

13. Fleming JO, Stohlman SA, Harmon RC, Lai MMC, Frelinger JA, Weiner LP (1983) Antigenic relationships of murine coronaviruses: analysis using monoclonal antibodies to JHM (MHV-4) virus. Virology 131: 296-307

14. Garlinghouse LE, Smith AL (1985) Responses of mice susceptible or resistant to lethal infection with mouse hepatitis virus, strain JHM, after exposure by a natural route. Lab Anim Sci 35: 469-472

15. Hierholzer JC, Broderson JR, Murphy FA (1979) New strain of mouse hepatitis virus as the cause of lethal enteritis in infant mice. Infect Immun 24: 508-522

16. Holmes KV (1986) Replication of coronaviruses. In: Fields BN, Knipe DM, Chanock RM, Melnick JL, Roizman B, Shope RE (eds) Fundamental virology. Raven Press, New York, pp 513-525

17. Keck JG, Makino S, Soe LH, Fleming JO, Stohlman SA, Lai MMC (1987) RNA recombination of coronavirus. Adv Exp Med Biol 218: 99-107

18. Nakanaga K, Yamanouchi K, Fujiwara K (1986) Protective effect of monoclonal antibodies on lethal mouse hepatitis virus infection in mice. J Virol 59: 168-171

19. Smith AL (1983) An immunofluorescence test for detection of serum antibody to rodent coronaviruses. Lab Anim Sci 33: 157-160

20. Smith AL, Barthold SW, Beck DS (1987) Intranasally administered alpha/beta interferon prevents extension of mouse hepatitis virus, strain JHM, into the brains of BALB/ cByJ mice. Antiviral Res 8: 239-246

21. Smith AL, Winograd DF (1986) Two enzyme immunoassays for detection of antibody to rodent coronaviruses. J Virol Methods 14: 335-343 
196 S. W. Barthold and Abigail L. Smith: Challenge immunity to coronavirus

22. Taguchi F, Goto Y, Aiuchi M, Hayashi T, Fujiwara K (1979) Pathogenesis of mouse hepatitis virus infection. The role of nasal epithelial cells as a primary target of lowvirulence virus, MHV-S. Microbiol Immunol 23: 249-262

23. Taguchi F, Yamada A, Fujiwara K (1980) Resistance to highly virulent mouse hepatitis virus acquired by mice after low-virulence infection: enhanced antiviral activity of macrophages. Infect Immun 29: 42-49

24. Talbot PJ, Buchmeier MJ (1985) Antigenic variation among murine coronaviruses: evidence for polymorphism on the peplomer glycoprotein, E 2. Virus Res $2: 317-328$

25. Wege H, Siddell S, ter Meulen V (1982) The biology and pathogenesis of coronaviruses. Curr Top Microbiol Immunol 99: 165-200

Authors' address: Dr. Stephen W. Barthold, Section of Comparative Medicine, Yale University School of Medicine, 333 Cedar Street, P.O. Box 3333, New Haven, CT 06510 , U.S.A.

Received November 25, 1988 